\title{
MOTION WITH FRICTION OF A HEAVY PARTICLE ON A MANIFOLD APPLICATIONS TO OPTIMIZATION
}

\begin{abstract}
Alexandre $\mathrm{CABOT}^{1}$
Abstract. Let $\Phi: H \rightarrow \mathbf{R}$ be a $\mathcal{C}^{2}$ function on a real Hilbert space and $\Sigma \subset H \times \mathbf{R}$ the manifold defined by $\Sigma:=$ Graph $(\Phi)$. We study the motion of a material point with unit mass, subjected to stay on $\Sigma$ and which moves under the action of the gravity force (characterized by $g>0$ ), the reaction force and the friction force $(\gamma>0$ is the friction parameter). For any initial conditions at time $t=0$, we prove the existence of a trajectory $x($.$) defined on \mathbf{R}_{+}$. We are then interested in the asymptotic behaviour of the trajectories when $t \rightarrow+\infty$. More precisely, we prove the weak convergence of the trajectories when $\Phi$ is convex. When $\Phi$ admits a strong minimum, we show moreover that the mechanical energy exponentially decreases to its minimum.
\end{abstract}

Mathematics Subject Classification. 34A12, 34G20, 37N40, 70Fxx.

Received: October 8, 2001. Revised: February 27, 2002.

\section{INTRODUCTION}

Let $H$ be a real Hilbert space, with scalar product and corresponding norm respectively denoted by $\langle.,$.$\rangle and$ |. . Let $\Phi: H \rightarrow \mathbf{R}$ be a function of class $\mathcal{C}^{2}$ and $\Sigma \subset H \times \mathbf{R}$ the manifold defined by $\Sigma:=$ Graph $(\Phi)$. In this paper, we study the motion of a material point with unit mass, subjected to stay on $\Sigma$ and which moves under the action of the gravity force (characterized by $g>0$ ), the reaction force and the friction force $(\gamma>0$ is the friction parameter). A simple application of the Fundamental Principle of Dynamics leads to the system

$$
(M P M) \quad \ddot{x}(t)+\gamma \dot{x}(t)+\frac{g+\left\langle\nabla^{2} \Phi(x(t)) \cdot \dot{x}(t), \dot{x}(t)\right\rangle}{1+|\nabla \Phi(x(t))|^{2}} \nabla \Phi(x(t))=0
$$

$((M P M)$ means "Mechanical Particle Motion"). We know experimentally that, because of the friction, the $(M P M)$ system is dissipative and the trajectory $x($.$) tends toward a local minimum of \Phi$. Moreover the velocity $\dot{x}($.$) and the gradient \nabla \Phi(x()$.$) tend to zero. The (M P M)$ system can be studied in the classical framework of the theory of dissipative systems (see, for example, Hale [5] and Haraux [6]).

Keywords and phrases. Mechanics of particles, dissipative dynamical system, optimization, convex minimization, asymptotic behaviour, gradient system, heavy ball with friction.

1 ACSIOM, CNRS-FRE 2311, Université Montpellier 2, place Eugène Bataillon, 34095 Montpellier Cedex 5, France.

e-mail: cabot@math.univ-montp2.fr 
The main interest in Optimization of the $(M P M)$ system is that it gives a mechanical interpretation of the classical Heavy Ball with Friction system

$$
(H B F) \quad \ddot{x}(t)+\gamma \dot{x}(t)+\nabla \Phi(x(t))=0 \quad(\gamma>0) .
$$

Indeed, since we are interested in the asymptotic behaviour of $x($.$) , we notice that \nabla \Phi(x)$ and $\left\langle\nabla^{2} \Phi(x) . \dot{x}, \dot{x}\right\rangle$ are negligible and $(M P M)$ then reduces to $(H B F)$. It is worth pointing out that, in a series of recent papers, most of the convergence results known in the case of the steepest descent, have been proved to be also valid in the case of the $(H B F)$ system. To quote only some of them, when $\Phi$ is convex, Bruck's theorem [4] known for the steepest descent, has been extended by Alvarez [1] to the case of the $(H B F)$ system. Under additionnal assumptions (for example, if $\Phi$ admits a strong minimum), Bolte [3] has obtained the exponential decay of the energy associated to $(H B F)$.

Our main purpose in this paper is to extend well-known results for the $(H B F)$ system ( $c f$. Attouch-GoudouRedont [2]) to the (MPM) system: we then rediscover some results suggested by our mechanical intuition. We stress the fact that, even if these results are intuitive from a mechanical point of view, their mathematical study is quite involved.

The paper is organized as follows. In Section 1, we precisely set the mechanical problem and we show how the F.P.D. leads to the $(M P M)$ system. In Section 2, we establish some global existence results (Th. 2.1). In Section 3, we are interested in the asymptotic behaviour of the trajectories when the potential $\Phi$ is convex. We first have a general result of weak convergence (Th. 3.1) and then, by reinforcing the assumptions on $\Phi$, we obtain the exponential decay of the energy along the trajectories (Th. 3.4).

\section{The Mechanical PROBlem}

Let us consider a real Hilbert space $H$, with scalar product $\langle.,$.$\rangle and a mapping \Phi: H \rightarrow \mathbf{R}$, of class $C^{2}$. Given a material point $M$ of mass $m$ which moves on the manifold defined by $\Sigma:=\operatorname{Graph}(\Phi), r(t) \in H \times \mathbf{R}$ denotes the position of $M$ at time $t$ :

$$
r(t)=\left(\begin{array}{c}
x(t) \\
\Phi(x(t))
\end{array}\right) \quad \text { when } x(t) \in H
$$

According to the fundamental principle of the dynamics, F.P.D. in short, the motion of $M$ is governed by the equation:

$$
m \ddot{r}=\vec{G}+\vec{F}+\vec{R}
$$

the second member of this equality being the sum of the forces which are applied to $M$ :

- The gravity force $\vec{G}=\left(\begin{array}{c}0 \\ -m g\end{array}\right)$.

- A force of friction of viscous type: this force is opposed to the movement of the particle (friction), and is proportional to the speed (viscous friction): $\vec{F}=-\lambda_{f} \dot{r}(t)$, where $\lambda_{f}>0$ is the friction coefficient.

- The reaction $\vec{R}$ of the surface $(\Sigma)$, which expresses that the particle does not penetrate into $(\Sigma): \vec{R}=R \vec{n}$ where $\vec{n}$ is the outwards unitary normal to $(\Sigma)$ at $M$.

Classically, $\vec{n}(x)=\frac{1}{\sqrt{1+|\nabla \Phi(x)|^{2}}}\left(\begin{array}{c}-\nabla \Phi(x) \\ 1\end{array}\right)$. Let us project (1) on $H$

$$
m \ddot{x}=-\lambda_{f} \dot{x}-\frac{R}{\sqrt{1+|\nabla \Phi(x)|^{2}}} \nabla \Phi(x) .
$$




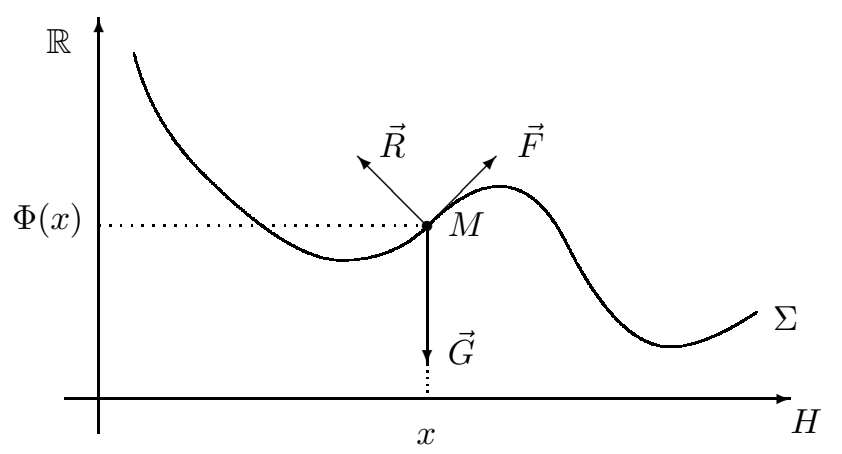

Figure 1.

We still have to determine the amplitude $R$ of the reaction of the surface $(\Sigma)$. For that purpose, let us project (1) on $\vec{n}$

$$
m\langle\ddot{r}, \vec{n}\rangle=\langle\vec{G}, \vec{n}\rangle+\langle\vec{F}, \vec{n}\rangle+\langle\vec{R}, \vec{n}\rangle
$$

Since $\langle\vec{F}, \vec{n}\rangle=0$ and $\langle\vec{R}, \vec{n}\rangle=R$, the previous expression becomes

$$
m\langle\ddot{r}, \vec{n}\rangle=-\frac{m g}{\sqrt{1+|\nabla \Phi(x)|^{2}}}+R
$$

An easy computation shows that

$$
\dot{r}(t)=\left(\begin{array}{c}
\dot{x}(t) \\
\langle\nabla \Phi(x(t)), \dot{x}(t)\rangle
\end{array}\right), \quad \ddot{r}(t)=\left(\begin{array}{c}
\ddot{x}(t) \\
\left\langle\nabla^{2} \Phi(x(t)) \cdot \dot{x}(t), \dot{x}(t)\right\rangle+\langle\nabla \Phi(x(t)), \ddot{x}(t)\rangle
\end{array}\right)
$$

which implies

$$
\langle\ddot{r}, \vec{n}\rangle=\frac{\left\langle\nabla^{2} \Phi(x) \cdot \dot{x}, \dot{x}\right\rangle}{\sqrt{1+|\nabla \Phi(x)|^{2}}}
$$

Combining (3) and (4), we find $R=\frac{m}{\sqrt{1+|\nabla \Phi(x)|^{2}}}\left(g+\left\langle\nabla^{2} \Phi(x) \cdot \dot{x}, \dot{x}\right\rangle\right)$. Injecting this expression of $R$ in equation (2), we obtain

$$
m \ddot{x}+\lambda_{f} \dot{x}+\frac{m}{1+|\nabla \Phi(x)|^{2}}\left(g+\left\langle\nabla^{2} \Phi(x) \cdot \dot{x}, \dot{x}\right\rangle\right) \nabla \Phi(x)=0 .
$$

Let us now divide by $m$ and define $\gamma=\lambda_{f} / m$ : we finally obtain the $(M P M)$ system

$$
(M P M) \quad \ddot{x}+\gamma \dot{x}+\frac{\left(g+\left\langle\nabla^{2} \Phi(x) \cdot \dot{x}, \dot{x}\right\rangle\right)}{1+|\nabla \Phi(x)|^{2}} \nabla \Phi(x)=0
$$

("Mechanical Particle Motion"). Let us notice that the "Heavy Ball with Friction" system

$$
(H B F) \quad \ddot{x}+\gamma \dot{x}+g \nabla \Phi(x)=0
$$


is an approximation of the $(M P M)$ system, where one considers that

- $|\nabla \Phi(x)|$ is negligible with respect to 1 .

- $\left\langle\nabla^{2} \Phi(x) \cdot \dot{x}, \dot{x}\right\rangle$ is negligible with respect to $g$.

The main key tool for studying $(M P M)$ is the existence of a natural Lyapounov function: the mechanical energy $E(t)=\frac{1}{2}|\dot{r}(t)|^{2}+g \Phi(x(t))$.

\section{Global existence}

Let $H$ be a real Hilbert space. Let us consider a mapping $\Phi: H \rightarrow \mathbf{R}$ which satisfies the following conditions $(\mathcal{H})\left\{\begin{array}{l}\Phi \text { is of class } \mathcal{C}^{2} \text { on } H \\ \Phi \text { is bounded from below on } H \\ \nabla^{2} \Phi \text { is Lipschitz continuous on the bounded subsets of } H .\end{array}\right.$

Let us consider the second order system in $H$

$$
(M P M) \quad \ddot{x}+\gamma \dot{x}+\frac{\left(g+\left\langle\nabla^{2} \Phi(x) \cdot \dot{x}, \dot{x}\right\rangle\right)}{1+|\nabla \Phi(x)|^{2}} \nabla \Phi(x)=0 .
$$

We can define along every trajectory of $(M P M)$ its mechanical energy by

$$
E(t)=\frac{1}{2}\left(|\dot{x}(t)|^{2}+\langle\nabla \Phi(x(t)), \dot{x}(t)\rangle^{2}\right)+g \Phi(x(t)) .
$$

The central result of this section is given by the following theorem.

Theorem 2.1. Let us assume that $\Phi: H \rightarrow \mathbf{R}$ satisfies the assumptions $(\mathcal{H})$ and that the parameters $\gamma$ and $g$ are positive. Then, the following properties hold:

(i) for all $\left(x_{0}, \dot{x}_{0}\right)$ in $H \times H$, there exists a unique solution $x($.$) of (M P M)$ defined on the whole interval $\left[0,+\infty\left[\right.\right.$, which is of class $\mathcal{C}^{2}$ on $\left[0,+\infty\left[\right.\right.$, and which satisfies the initial conditions $x(0)=x_{0}$ and $\dot{x}(0)=\dot{x}_{0}$.

(ii) for every trajectory $x($.$) of (M P M)$, the corresponding energy $E(t)$ is decreasing on $[0,+\infty[$ and bounded from below, and hence converges to some real value $E_{\infty}$. Moreover,

$$
\dot{x} \in L^{\infty}(0,+\infty ; H) \cap L^{2}(0,+\infty ; H) .
$$

(iii) Assuming moreover that $x($.$) is bounded, then we have$

- $\lim _{t \rightarrow+\infty} \dot{x}(t)=0$ and $\lim _{t \rightarrow+\infty} \ddot{x}(t)=0$,

- $\lim _{t \rightarrow+\infty} \nabla \Phi(x(t))=0$ and $\lim _{t \rightarrow+\infty} \Phi(x(t))=\frac{E_{\infty}}{g}$.

Proof of Theorem 2.1.

Proof of (i). The system $(M P M)$ can be written as a first order system in $H \times H$ :

$$
\dot{Y}=F(Y)
$$

with

$$
Y(t)=(x(t), \dot{x}(t))^{t} \quad \text { and } \quad F(u, v)=\left(v,-\gamma v-\frac{\left(g+\left\langle\nabla^{2} \Phi(u) \cdot v, v\right\rangle\right)}{1+|\nabla \Phi(u)|^{2}} \nabla \Phi(u)\right)^{t}
$$


For $Y_{0}=\left(x_{0}, \dot{x}_{0}\right)^{t}$ given in $H \times H$, the Cauchy-Lipschitz theorem and hypothesis $(\mathcal{H})$ ensure the existence of a unique local solution to the problem:

$$
\left\{\begin{array}{l}
\dot{Y}=F(Y) \\
Y(0)=Y_{0}
\end{array}\right.
$$

Let $x($.$) denote the corresponding maximal solution which is defined on some interval \left[0, T_{\max }\left[\right.\right.$ with $0<T_{\max } \leq$ $+\infty$. In order to prove that $T_{\max }=+\infty$, let us show that $\dot{x}($.$) is bounded.$

We first observe that equation $(M P M)$ and the regularity assumptions on $\Phi$ automatically imply that $x($. is $\mathcal{C}^{2}$ on $\left[0, T_{\max }[\right.$. By differentiation of $E(t)$, we obtain

$$
\begin{aligned}
\dot{E}(t) & =\langle\dot{x}, \ddot{x}\rangle+\langle\nabla \Phi(x), \dot{x}\rangle\langle\nabla \Phi(x), \ddot{x}\rangle+\langle\nabla \Phi(x), \dot{x}\rangle\left\langle\nabla^{2} \Phi(x) . \dot{x}, \dot{x}\right\rangle+g\langle\nabla \Phi(x), \dot{x}\rangle \\
& =\langle\ddot{x}, \dot{x}+\langle\nabla \Phi(x), \dot{x}\rangle \nabla \Phi(x)\rangle+\langle\nabla \Phi(x), \dot{x}\rangle\left\langle\nabla^{2} \Phi(x) \cdot \dot{x}, \dot{x}\right\rangle+g\langle\nabla \Phi(x), \dot{x}\rangle .
\end{aligned}
$$

Since $\ddot{x}=-\gamma \dot{x}-\frac{\left(g+\left\langle\nabla^{2} \Phi(x) \cdot \dot{x}, \dot{x}\right\rangle\right)}{1+|\nabla \Phi(x)|^{2}} \nabla \Phi(x)$, one has

$$
\begin{aligned}
\dot{E}(t)= & -\gamma\left(|\dot{x}|^{2}+\langle\nabla \Phi(x), \dot{x}\rangle^{2}\right)-\frac{\left(g+\left\langle\nabla^{2} \Phi(x) \cdot \dot{x}, \dot{x}\right\rangle\right)}{1+|\nabla \Phi(x)|^{2}}\langle\nabla \Phi(x), \dot{x}\rangle\left(1+|\nabla \Phi(x)|^{2}\right) \\
& +\langle\nabla \Phi(x), \dot{x}\rangle\left\langle\nabla^{2} \Phi(x) \cdot \dot{x}, \dot{x}\right\rangle+g\langle\nabla \Phi(x), \dot{x}\rangle .
\end{aligned}
$$

After simplifications, we find

$$
\dot{E}(t)=-\gamma\left(|\dot{x}|^{2}+\langle\nabla \Phi(x), \dot{x}\rangle^{2}\right) .
$$

Thus, the function $E($.$) is decreasing and for all t \in\left[0, T_{\max }[, E(t) \leq E(0)\right.$. Equivalently,

$$
\frac{1}{2}|\dot{x}(t)|^{2}+\frac{1}{2}\langle\nabla \Phi(x(t)), \dot{x}(t)\rangle^{2}+g \Phi(x(t)) \leq E(0) .
$$

Since $\Phi$ is bounded from below, we obtain that

$$
\sup _{t \in\left[0, T_{\max }[\right.}|\dot{x}(t)|:=C<+\infty .
$$

It is a standard argument to derive from such an estimation, that $T_{\max }=+\infty$.

Indeed, let us argue by contradiction, and assume that $T_{\max }<+\infty$. We have

$$
\left|x(t)-x\left(t^{\prime}\right)\right| \leq C\left|t-t^{\prime}\right|,
$$

and since $T_{\max }<+\infty, \lim _{t \rightarrow T_{\max }} x(t):=x_{\infty}$ exists. So, $x($.$) and \dot{x}($.$) are bounded on \left[0, T_{\max }[\right.$, and by equation $(M P M), \ddot{x}($.$) is bounded too on this interval. So \lim _{t \rightarrow T_{\max }} \dot{x}(t)=\dot{x}_{\infty}$ exists. But, applying again the local existence theorem with initial data $\left(x_{\infty}, \dot{x}_{\infty}\right)$, we can extend the maximal solution to a strictly larger interval, which is a clear contradiction. So, $T_{\max }=+\infty$, which completes the proof of (i).

Proof of (ii). We already proved that $E($.$) is decreasing. Since \Phi$ is bounded from below, and since $E(t) \geq$ $g \Phi(x(t))$, the energy $E($.$) is also bounded from below. As a consequence, \lim _{t \rightarrow+\infty} E(t)=E_{\infty}$ exists, with $E_{\infty} \in \mathbf{R}$. Using (8), and the fact that $\Phi$ is bounded from below, we obtain that, for all $t \geq 0$

$$
\frac{1}{2}|\dot{x}(t)|^{2} \leq E(0)-g \inf \Phi .
$$


Hence,

$$
\dot{x} \in L^{\infty}(0,+\infty ; H)
$$

From (7), we derive that, for all $0 \leq t<+\infty$

$$
\int_{0}^{t}|\dot{x}(s)|^{2} \mathrm{~d} s \leq \frac{1}{\gamma}(E(0)-E(t))
$$

Since $E(t)$ decreases to $E_{\infty}$ as $t$ increases to $+\infty$, we obtain that

$$
\int_{0}^{+\infty}|\dot{x}(s)|^{2} \mathrm{~d} s \leq \frac{1}{\gamma}\left(E(0)-E_{\infty}\right)
$$

and $\dot{x} \in L^{2}(0,+\infty ; H)$.

Proof of (iii). We now assume that $x$ is bounded. We have proved in (ii) that $\dot{x}$ is bounded; therefore equation $(M P M)$ and the fact that $\nabla \Phi$ and $\nabla^{2} \Phi$ are bounded on the bounded subsets of $H$ imply that $\ddot{x}$ is bounded.

Let us now observe that the function $h(t):=\dot{x}(t)$ satisfies both

$$
h \in L^{2}(0,+\infty ; H) \quad \text { and } \quad \dot{h} \in L^{\infty}(0,+\infty ; H) .
$$

According to a classical result, these two properties imply: $\lim _{t \rightarrow+\infty} h(t)=0$.

Let us now prove that $\lim _{t \rightarrow+\infty} \ddot{x}(t)=0$. Let us first prove this result in the simpler case where $\Phi$ is of class $\mathcal{C}^{3}$, then we shall see how one can adapt this argument when $\Phi$ is only of class $\mathcal{C}^{2}$.

Returning to equation $(M P M)$, since $\Phi$ is $\mathcal{C}^{3}$, the solution $x$ is $\mathcal{C}^{3}$. By differentiating the equation we obtain

$$
\dddot{x}+\gamma \ddot{x}=f(t)
$$

with

$$
f(t)=-\frac{\mathrm{d}}{\mathrm{d} t}\left[\frac{\left(g+\left\langle\nabla^{2} \Phi(x) \cdot \dot{x}, \dot{x}\right\rangle\right)}{1+|\nabla \Phi(x)|^{2}} \nabla \Phi(x)\right] .
$$

Since, by assumption, $\nabla \Phi, \nabla^{2} \Phi, \nabla^{3} \Phi$ are bounded on the bounded subsets and since $x, \dot{x}$ and $\ddot{x}$ are bounded, one can easily verify by differentiating $f$ that there exists $C \geq 0$ such that $|f(t)| \leq C|\dot{x}(t)|$ for all $t \geq 0$. Using now $\lim _{t \rightarrow+\infty} \dot{x}(t)=0$, we finally obtain

$$
\lim _{t \rightarrow+\infty} f(t)=0
$$

If we set $z=\ddot{x}$, equation (9) becomes: $\dot{z}+\gamma z=f$. After integration of this equation it is easy to verify that (10) implies convergence of $z(t)=\ddot{x}(t)$ to 0 as $t \rightarrow+\infty$.

When $\Phi$ is not $\mathcal{C}^{3}$, we have to adapt the preceding argument. The idea is to replace the derivative $\dddot{x}$, which a priori makes no sense, by a differential quotient. We refer to Attouch-Goudou-Redont [2], proof of Theorem 3.1. Since $\lim _{t \rightarrow+\infty} \dot{x}(t)=\lim _{t \rightarrow+\infty} \ddot{x}(t)=0$, it is now clear in view of $(M P M)$ that $\lim _{t \rightarrow+\infty} \nabla \Phi(x(t))=0$.

We complete the proof of (iii) by noticing that, since

$$
E(t)=\frac{1}{2}|\dot{x}(t)|^{2}+\frac{1}{2}\left\langle\nabla \Phi(x(t), \dot{x}(t)\rangle^{2}+g \Phi(x(t)) \rightarrow E_{\infty} \quad \text { as } t \rightarrow+\infty\right.
$$




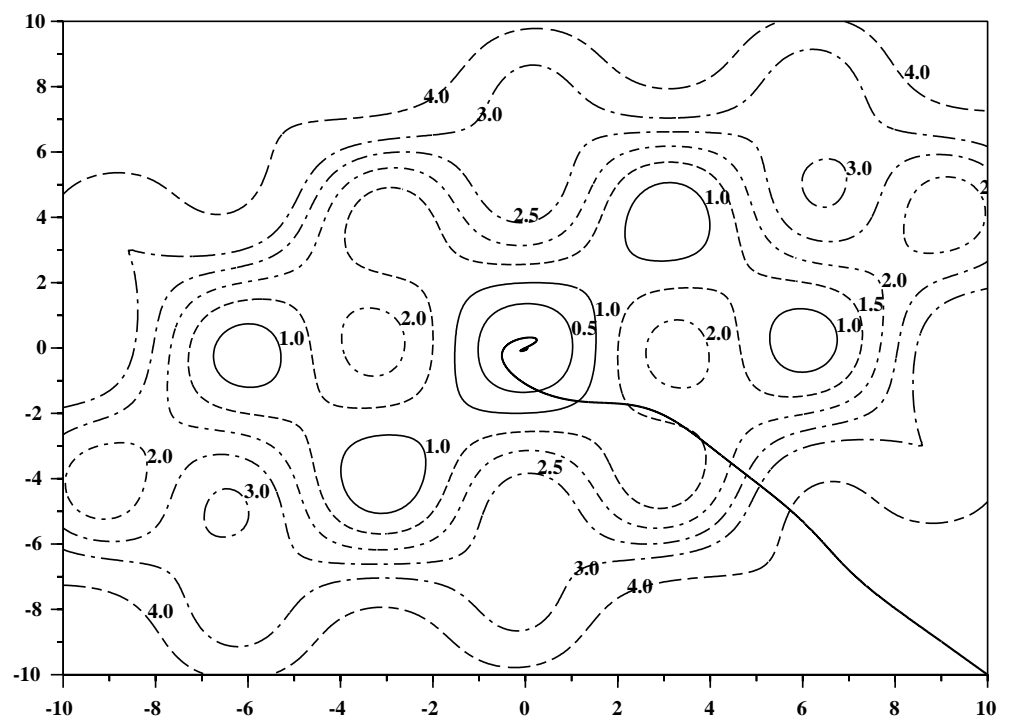

Figure 2. A trajectory of the $(M P M)$ system with $\Phi\left(x_{1}, x_{2}\right)=\left(2 x_{1}^{2}+x_{2}^{2}-x_{1} x_{2}\right) / 50-$ $\cos x_{1} \cos \frac{x_{2}}{\sqrt{2}}+1, x_{0}=(10,-10), \dot{x}_{0}=(-5,5), \gamma=1, g=1$.

and since $\dot{x}(t) \rightarrow 0$ as $t \rightarrow+\infty$, we have that

$$
\lim _{t \rightarrow+\infty} \Phi(x(t))=\frac{E_{\infty}}{g} .
$$

Corollary 2.2. Under the hypotheses of Theorem 2.1, additionally assume that $\Phi$ is coercive, i.e. $\lim _{|x| \rightarrow+\infty} \Phi(x)=$ $+\infty$. Then $x$ is bounded and the conclusions of Theorem 2.1 hold.

Proof of Corollary 2.2. It is enough to observe that inequality (8) gives

$$
\Phi(x(t)) \leq \frac{E(0)}{g} .
$$

This majorization on $\Phi(x(t))$ and the coerciveness of $\Phi$ imply that the trajectory $x($.$) remains bounded.$

Remark. When there is no effect of gravity $(g=0)$, the situation is quite different from the previous one. The $(M P M)$ system then reduces to

$$
\ddot{x}+\gamma \dot{x}+\frac{\left\langle\nabla^{2} \Phi(x) \cdot \dot{x}, \dot{x}\right\rangle}{1+|\nabla \Phi(x)|^{2}} \nabla \Phi(x)=0,
$$

and the energy function $E(t)=\frac{1}{2}\left(|\dot{x}(t)|^{2}+\langle\nabla \Phi(x(t)), \dot{x}(t)\rangle^{2}\right)$ then verifies the following decay law

$$
\dot{E}(t)=-\gamma\left(|\dot{x}(t)|^{2}+\langle\nabla \Phi(x(t)), \dot{x}(t)\rangle^{2}\right)=-2 \gamma E(t)
$$

Consequently, the energy $E$ exponentially decreases to $0: E(t)=E(0) \mathrm{e}^{-2 \gamma t}$ and in particular

$$
|\dot{x}(t)| \leq \sqrt{2 E(0)} \mathrm{e}^{-\gamma t}
$$


Then, we obviously have $|\dot{x}| \in L^{1}(0,+\infty)$ and there exists some $x_{\infty} \in H$ such that the trajectory $x($.$) strongly$ converges to $x_{\infty}$. Let us note that, in general, when $g=0$, we do not have $\lim _{t \rightarrow+\infty} \nabla \Phi(x(t))=0$ and a fortiori $\nabla \Phi\left(x_{\infty}\right) \neq 0$.

\section{Asymptotic Behaviour in the CONVEX CASE}

\subsection{A general result of weak convergence}

In this section, $H$ is a Hilbert space, $\Phi: H \rightarrow \mathbf{R}$ is a convex function which is $\mathcal{C}^{2}$, with $\nabla^{2} \Phi$ Lipschitz continuous on the bounded sets of $H$, and which satisfies: $\Phi$ is bounded from below and $S=\operatorname{argmin} \Phi \neq \emptyset$.

Alvarez has proved in [1] that each trajectory of the $(H B F)$ system, in the convex case, weakly converges to a global minimum of $\Phi$. We have the same result for the $(M P M)$ system.

Theorem 3.1. Let us assume that $\Phi: H \rightarrow \mathbf{R}$ satisfies the assumptions $(\mathcal{H})$. We additionnaly assume that $\Phi$ is convex and argmin $\Phi \neq \emptyset$. For all $x_{0} \in H, \dot{x}_{0} \in H$, let $x$ be the unique solution of the (MPM) system

$$
\left\{\begin{array}{l}
\ddot{x}+\gamma \dot{x}+\frac{\left(g+\left\langle\nabla^{2} \Phi(x) \cdot \dot{x}, \dot{x}\right\rangle\right)}{1+|\nabla \Phi(x)|^{2}} \nabla \Phi(x)=0 \\
x(0)=x_{0}, \dot{x}(0)=\dot{x}_{0} .
\end{array}\right.
$$

Then, we have

(a) Estimations of the energy decay:

(i) $E()-.g \min \Phi \in L^{1}(0,+\infty)$.

(ii) $\lim _{t \rightarrow+\infty} t(E(t)-g \min \Phi)=0$ and as a consequence

$$
\lim _{t \rightarrow+\infty} t(\Phi(x(t))-\min \Phi)=0 \quad \text { and } \quad \lim _{t \rightarrow+\infty} t|\dot{x}(t)|^{2}=0 .
$$

(b) Convergence of the trajectory: there exists $x_{\infty} \in \operatorname{argmin} \Phi$ such that $x(t) \rightarrow x_{\infty}$ weakly in $H$ as $t \rightarrow+\infty$.

Proof of Theorem 3.1.

Proof of $(a)-(i)$. Like in the study of the $(H B F)$ system ( $c f$. Alvarez [1]), we introduce the function $h(t):=\frac{1}{2}|x(t)-z|^{2}$, where $z \in \operatorname{argmin} \Phi$. Let us set

$$
k(t)=\dot{h}(t)+\gamma h(t)=\langle\dot{x}(t), x(t)-z\rangle+\frac{\gamma}{2}|x(t)-z|^{2} .
$$

The functions $h$ and $k$ are closely linked because, as we will see later, $\dot{h}$ vanishes at infinity. A simple computation yields

$$
\dot{k}(t)=|\dot{x}(t)|^{2}+\langle\ddot{x}(t)+\gamma \dot{x}(t), x(t)-z\rangle .
$$

Since $x($.$) is solution of (M P M)$,

$$
\dot{k}(t)=|\dot{x}(t)|^{2}-\frac{\left(g+\left\langle\nabla^{2} \Phi(x(t)) \cdot \dot{x}(t), \dot{x}(t)\right\rangle\right)}{1+|\nabla \Phi(x(t))|^{2}}\langle\nabla \Phi(x(t)), x(t)-z\rangle .
$$

By convexity of $\Phi$,

$$
\Phi(z)-\Phi(x(t)) \geq\langle\nabla \Phi(x(t)), z-x(t)\rangle
$$


and

$$
\left\langle\nabla^{2} \Phi(x(t)) \cdot \dot{x}(t), \dot{x}(t)\right\rangle \geq 0
$$

which yields

$$
\dot{k}(t)+\frac{g}{1+|\nabla \Phi(x(t))|^{2}}(\Phi(x(t))-\min \Phi) \leq|\dot{x}(t)|^{2} .
$$

In particular, we have $\dot{k}(t) \leq|\dot{x}(t)|^{2}$, i.e. $\dot{k}_{+} \in L^{1}(0,+\infty)$. Since $k$ is bounded from below, $\lim _{t \rightarrow+\infty} k(t)$ exists and hence the map $x$ is bounded. Since $\nabla \Phi$ is bounded on the bounded sets of $\mathrm{H}$, there exists $M \geq 0$ such that $|\nabla \Phi(x(t))| \leq M$ for all $t \geq 0$. Setting $C=\frac{1}{1+M^{2}}$ and recalling that $E=\frac{1}{2}\left(|\dot{x}|^{2}+\langle\nabla \Phi(x), \dot{x}\rangle^{2}\right)+g \Phi(x),(11)$ gives

$$
\dot{k}(t)+C(E(t)-g \min \Phi) \leq \frac{3}{2}|\dot{x}(t)|^{2}
$$

Let us now integrate $(12)$ on $[0, t]$

$$
k(t)-k(0)+C \int_{0}^{t}(E(s)-g \min \Phi) \mathrm{d} s \leq \frac{3}{2} \int_{0}^{t}|\dot{x}(s)|^{2} \mathrm{~d} s .
$$

Passing to the limit when $t \rightarrow+\infty$, we find clearly $E()-.g \min \Phi \in L^{1}(0,+\infty)$.

Proof of $(a)-(i i)$. Since $E($.$) is non increasing,$

$$
\int_{\frac{t}{2}}^{t}(E(s)-g \min \Phi) \mathrm{d} s \geq \frac{t}{2}(E(t)-g \min \Phi) \geq 0 .
$$

Passing now to the limit when $t \rightarrow+\infty$, one obtains $\lim _{t \rightarrow+\infty} t(E(t)-g \min \Phi)=0$. The rest of assertion (a) $-($ ii $)$ is straightforward.

Proof of $(b)$. The technique which is used here to prove the convergence of the trajectory has already been used by Alvarez in [1]. In particular, the Opial lemma plays a central role. For the convenience of the reader, we recall it.

Lemma 3.2 (Opial). Let $H$ be a Hilbert space and $x:[0,+\infty[\rightarrow H$ be a function such that there exists a non void set $S \subset H$ which verifies:

(i) $\forall t_{n} \rightarrow+\infty$ with $x\left(t_{n}\right) \rightarrow x_{\infty}$ weakly in $H$, we have $x_{\infty} \in S$.

(ii) $\forall z \in S, \lim _{t \rightarrow+\infty}|x(t)-z|$ exists.

Then, $x(t)$ weakly converges as $t \rightarrow+\infty$ to some element $x_{\infty}$ of $S$.

We are now going to apply the Opial lemma with $S=\operatorname{argmin} \Phi \neq \emptyset$. We have to check points (i) and (ii) of Lemma 3.2.

- Let us assume that $w-\lim _{n \rightarrow+\infty} x\left(t_{n}\right)=x_{\infty}$. We then have $\liminf _{n \rightarrow+\infty} \Phi\left(x\left(t_{n}\right)\right) \geq \Phi\left(x_{\infty}\right)$ because $\Phi$ is convex and continuous, hence lower semicontinuous for the weak topology. But, in view of $(a)-(i i)$, $\lim _{t_{n} \rightarrow+\infty} \Phi\left(x\left(t_{n}\right)\right)=\min \Phi$. Hence $\Phi\left(x_{\infty}\right) \leq \min \Phi$ and therefore $x_{\infty} \in \operatorname{argmin} \Phi$.

- Let us now prove that $\lim _{t \rightarrow+\infty}|x(t)-z|$ exists. We know that

$$
\lim _{t \rightarrow+\infty} k(t)=\lim _{t \rightarrow+\infty}\left[\langle\dot{x}(t), x(t)-z\rangle+\frac{\gamma}{2}|x(t)-z|^{2}\right] \quad \text { exists. }
$$


On the other hand, $x($.$) is bounded and from (a)-(i i), \lim _{t \rightarrow+\infty} \dot{x}(t)=0$. Consequently $\lim _{t \rightarrow+\infty}\langle\dot{x}(t), x(t)-$ $z\rangle=0$ and hence, with $(13), \lim _{t \rightarrow+\infty}|x(t)-z|^{2}$ exists. This is true for any $z \in \operatorname{argmin} \Phi$, and hence the Opial lemma applies: there exists $x_{\infty} \in \operatorname{argmin} \Phi$ such that

$$
w-\lim _{t \rightarrow+\infty} x(t)=x_{\infty}
$$

It is worth completing the previous theorem by a strong convergence result when $\Phi$ is strongly convex.

Proposition 3.3. In addition to the assumptions of Theorem 3.1, let us assume that $\Phi$ is strongly convex, that is, for any $R>0$, there exists a function $\beta_{R}: \mathbf{R}^{+} \rightarrow \mathbf{R}^{+}$with $\beta_{R}\left(t_{n}\right) \rightarrow 0 \Longrightarrow t_{n} \rightarrow 0$, such that

$\forall x, y \in H \quad$ with $|x|<R,|y|<R$,

$$
\langle\nabla \Phi(x)-\nabla \Phi(y), x-y\rangle \geq \beta_{R}(|x-y|) .
$$

Then each trajectory $x($.$) of the (M P M)$ system is norm convergent as $t$ goes to $+\infty$ to the unique global minimizer $\bar{x}$ of $\Phi$.

Proof of Proposition 3.3. Let us consider a trajectory $x($.$) of the (M P M)$ system. We already know that the trajectory is bounded. So, there exists some $R>0$ such that for all $t \in[0,+\infty[,|x(t)| \leq R$. Since $\Phi$ is strongly convex, it has a unique minimizer $\bar{x}$. Let us write the strong monotonicity property (14) at $\bar{x}$ and $x(t)$ :

$$
\langle\nabla \Phi(\bar{x})-\nabla \Phi(x(t)), \bar{x}-x(t)\rangle \geq \beta_{R}(|x(t)-\bar{x}|) .
$$

Since $\nabla \Phi(\bar{x})=0$, it follows that

$$
\beta_{R}(|x(t)-\bar{x}|) \leq|\nabla \Phi(x(t))||\bar{x}-x(t)|
$$

From Theorem 2.1, we have $\lim _{t \rightarrow+\infty} \nabla \Phi(x(t))=0$. Since $x($.$) is bounded, it follows from (15) that$ $\lim _{t \rightarrow+\infty} \beta_{R}(|x(t)-\bar{x}|)=0$. From this we deduce that $x(t) \rightarrow \bar{x}$ strongly as $t \rightarrow+\infty$.

\subsection{Exponential decay of the energy}

We have proved in the previous section that, for a convex function $\Phi$, the solution $x$ of the $(M P M)$ system weakly converges to some $x_{\infty}$. We are now going to improve this result when $\Phi$ admits a strong minimum. More precisely, under this condition, the energy exponentially decreases to its minimum and the trajectory of $(M P M)$ strongly converges to its limit. This result has already been proved by Bolte [3] for the $(H B F)$ system. We use here a quite similar technique.

Theorem 3.4. In addition to the assumptions of Theorem 3.1, let us assume that $a \in H$ is a strong minimum of $\Phi$, i.e. there exists some $\alpha>0$ such that

$$
\forall x \in H, \quad \Phi(x)-\Phi(a) \geq \alpha|x-a|^{2} .
$$

Let $x$ be the unique solution of the $(M P M)$ system and $E()=.\frac{1}{2}\left(|\dot{x}|^{2}+\langle\nabla \Phi(x), \dot{x}\rangle^{2}\right)+g \Phi(x)$ the associated energy function.

Then, the energy function $E($.$) exponentially decreases to g \min \Phi$, i.e. there exists some $C>0$ and $\delta>0$ such that

$$
0 \leq E(t)-g \min \Phi \leq C \mathrm{e}^{-\delta t}
$$

Moreover, the trajectory $x$ strongly converges to a and satisfies

$$
|x(t)-a|^{2} \leq \frac{C}{g \alpha} \mathrm{e}^{-\delta t} .
$$


Proof of Theorem 3.4. Let us recall that the energy function satisfies the following decay law

$$
\dot{E}(t)=-\gamma\left(|\dot{x}(t)|^{2}+\langle\nabla \Phi(x(t)), \dot{x}(t)\rangle^{2}\right) \leq-\gamma|\dot{x}(t)|^{2}
$$

Like in the proof of Theorem 3.1, we denote by $k$ the function

$$
k(t)=\langle\dot{x}(t), x(t)-a\rangle+\frac{\gamma}{2}|x(t)-a|^{2}
$$

Without any loss of generality, we can assume that $a=0$ and $\Phi(a)=0$. The function $k$ then reduces to $k(t)=\langle\dot{x}(t), x(t)\rangle+\frac{\gamma}{2}|x(t)|^{2}$. In view of (12), there exists $C>0$ such that

$$
\dot{k}(t)+C E(t) \leq \frac{3}{2}|\dot{x}(t)|^{2}
$$

Let us multiply this last inequality by $\left.\eta \in] 0, \frac{2 \gamma}{3}\right]$ and add to (16); we obtain

$$
\dot{E}(t)+\eta \dot{k}(t)+\eta C E(t) \leq 0
$$

We now need a claim.

Claim 3.5. There exists some $C_{1}>0$ such that

$$
\forall t \geq 0, \quad-\frac{1}{\gamma} E(t) \leq k(t) \leq C_{1} E(t)
$$

Proof of Claim 3.5. We first prove that $k(t) \leq C_{1} E(t)$ for some $C_{1}>0$. We classically have, for all $\theta>0$,

$$
k(t)=\langle\dot{x}(t), x(t)\rangle+\frac{\gamma}{2}|x(t)|^{2} \leq \frac{\theta}{2}|x(t)|^{2}+\frac{|\dot{x}(t)|^{2}}{2 \theta}+\frac{\gamma}{2}|x(t)|^{2} .
$$

Hence, using $\Phi(x) \geq \alpha|x|^{2}$,

$$
k(t) \leq \frac{\theta+\gamma}{2 \alpha} \Phi(x(t))+\frac{|\dot{x}(t)|^{2}}{2 \theta} .
$$

Since $E(t) \geq \frac{1}{2}|\dot{x}(t)|^{2}+g \Phi(x(t))$, we deduce

$$
k(t) \leq \frac{\theta+\gamma}{2 \alpha g} E(t)-|\dot{x}(t)|^{2}\left(\frac{\theta+\gamma}{4 \alpha g}-\frac{1}{2 \theta}\right) .
$$

We now choose $\theta$ sufficiently large to have $\frac{\theta+\gamma}{4 \alpha g} \geq \frac{1}{2 \theta}$. Setting then $C_{1}=\frac{\theta+\gamma}{2 \alpha g}$, we obtain the expected formula. Let us now prove that $k(t) \geq-\frac{1}{\gamma} E(t)$. From the inequality $\langle x(t), \dot{x}(t)\rangle \geq-\frac{\gamma}{2}|x(t)|^{2}-\frac{|\dot{x}(t)|^{2}}{2 \gamma}$, we immediately deduce

$$
k(t) \geq-\frac{|\dot{x}(t)|^{2}}{2 \gamma} \geq-\frac{E(t)}{\gamma}
$$

In view of the previous claim, for all $\lambda \in] 0,1[$,

$$
E(t)=\lambda E(t)+(1-\lambda) E(t) \geq \lambda E(t)+\frac{1-\lambda}{C_{1}} k(t)
$$


We choose $\lambda$ so as to verify $\frac{1-\lambda}{C_{1}}=\eta \lambda$. Then, we have $E(t) \geq \lambda(E(t)+\eta k(t))$ and therefore, in view of (18)

$$
\dot{E}(t)+\eta \dot{k}(t)+\eta C \lambda(E(t)+\eta k(t)) \leq 0 .
$$

Consequently, setting $\delta=\eta C \lambda$ and $C_{2}=E(0)+\eta k(0)$,

$$
E(t)+\eta k(t) \leq C_{2} \mathrm{e}^{-\delta t} .
$$

Using again Claim 3.5, we find $E(t)+\eta k(t) \geq\left(1-\frac{\eta}{\gamma}\right) E(t) \geq \frac{E(t)}{3}$ and finally

$$
0 \leq E(t) \leq 3 C_{2} \mathrm{e}^{-\delta t} .
$$

The rest of the theorem is a straightforward consequence of the previous inequality.

Further remarks - Other possible developments. We stress the fact that, from a numerical point of view, the system $(M P M)$ is not competitive because of the hessian matrix $\nabla^{2} \Phi$. Indeed this term is very heavy in computational time. From a numerical point of view, $(M P M)$ can advantageously been replaced by $(H B F)$, which is easier to handle.

In the whole paper, we have considered the motion of a material point M. It would be possible to study the case of a real heavy ball with a positive radius. The mechanical study would be much more complicated but it would perhaps make appear new interesting terms from the Optimization point of view.

\section{REFERENCES}

[1] F. Alvarez, On the minimizing property of a second order dissipative system in Hilbert space. SIAM J. Control Optim. 38 (2000) 1102-1119.

[2] H. Attouch, X. Goudou and P. Redont, The heavy ball with friction method. I The continuous dynamical system. Commun. Contemp. Math. 2 (2000) 1-34.

[3] J. Bolte, Exponential decay of the energy for a second-order in time dynamical system. Working paper, Département de Mathématiques, Université Montpellier II.

[4] R.E. Bruck, Asymptotic convergence of nonlinear contraction semigroups in Hilbert space. J. Funct. Anal. 18 (1975) 15-26.

[5] J.K. Hale, Asymptotic behavior of dissipative systems. Mathematical Surveys and Monographs, 25. American Mathematical Society, Providence, RI (1988).

[6] A. Haraux, Systèmes dynamiques dissipatifs et applications. RMA 17, Masson, Paris (1991).

[7] Z. Opial, Weak convergence of the sequence of successive approximations for nonexpansive mappings. Bull. Amer. Math. Soc. 73 (1967) 591-597. 